\section{Observational evidence for the accretion-disk origin for a radio jet in an active galaxy}

Alan P. Marscher ${ }^{\star}$, Svetlana G. Jorstad ${ }^{\star} \dagger$, José-Luis Gómez $\ddagger$, Margo F. Aller $\$$, Harri Teräsranta\|, Matthew L. Listerף \& Alastair M. Stirling\#

${ }^{*}$ Institute for Astrophysical Research, Boston University, 725 Commonwealth Avenue, Boston, Massachusetts 02215, USA

$\dagger$ Sobolev Astronomical Institute, St Petersburg State University, Universitetskii Prospekt 28, Petrodvorets, St Petersburg, 198504, Russia

$\ddagger$ Insituto de Astrofísica de Andalucía (CSIC), Apartado Correos 3004, Granada, E-18080, Spain

$\$$ Astronomy Department, University of Michigan, 830 Dennison, 501 East

University Street, Ann Arbor, Michigan 48109-1090, USA

|| Metsähovi Radio Observatory, Helsinki University of Technology, Metsähovintie 114, Kylmala, Finland-02540

I National Radio Astronomy Observatory, 520 Edgemont Road, Charlottesville, Virginia 22903-2475, USA

\# Center for Astrophysics, University of Central Lancashire, Preston, Lancashire, PR1 2HE, UK

Accretion of gas onto black holes is thought to power the relativistic jets of material ejected from active galactic nuclei (AGN) and the 'microquasars' located in our Galaxy ${ }^{1-3}$. In microquasars, superluminal radio-emitting features appear and propagate along the jet shortly after sudden decreases in the $\mathrm{X}$ ray fluxes ${ }^{1}$. This establishes a direct observational link between the black hole and the jet: the X-ray dip is probably caused by the disappearance of a section of the inner accretion disk ${ }^{4}$ as it falls past the event horizon, while the remainder of the disk section is ejected into the jet, creating the appearance of a superluminal bright spot $^{5}$. No such connection has hitherto been established for AGN, because of insufficient multi-frequency data. Here we report the results of three years of monitoring the $\mathrm{X}$-ray and radio emission of the galaxy $3 \mathrm{C} 120$. As has been observed for microquasars, we find that dips in the X-ray emission are followed by ejections of bright superluminal knots in the radio jet. The mean time between X-ray dips appears to scale roughly with the mass of the black hole, although there are at present only a few data points.

We selected 3C120 (redshift $z=0.033$ ) owing to the combination of superluminal motion in its radio jet ${ }^{6,7}$ and its Seyfert-like X-ray properties. The X-ray energy spectrum ${ }^{8-11}$ (flux density as a function of frequency) is well fitted by a power law of slope $\alpha_{\mathrm{E}}=-0.7$ to -1 , plus an iron emission line at a photon energy of $6.4 \mathrm{keV}$. The prevailing models ${ }^{11}$ explain the X-ray emission from Seyferts in a similar manner as that from microquasars ${ }^{4}$ : soft X-rays from the inner accretion disk plus harder X-rays from a corona of hot electrons that reprocess the disk radiation. The presence of the iron line implies that most of the X-ray flux arises from the region near the accretion disk rather than in the jet. The similarity between its X-ray emission and that of microquasars, plus the presence of superluminal motion in the radio jets of both, led us to search for a connection between X-ray events and superluminal ejections in 3C120.

We imaged the compact radio jet of 3C120 with the Very Long Baseline Array (VLBA) roughly monthly from November 1997 to March 1999 (refs 6, 7), and bimonthly thereafter. A description of the data analysis procedures is provided in refs 6 and 7. The sequence of images between March 1998 and April 2001 is shown in Fig. 1. Diagonal lines indicate the trajectories of all major superluminal knots that emerged from the core during the period of our X-ray monitoring programme. Each of these is distinguished

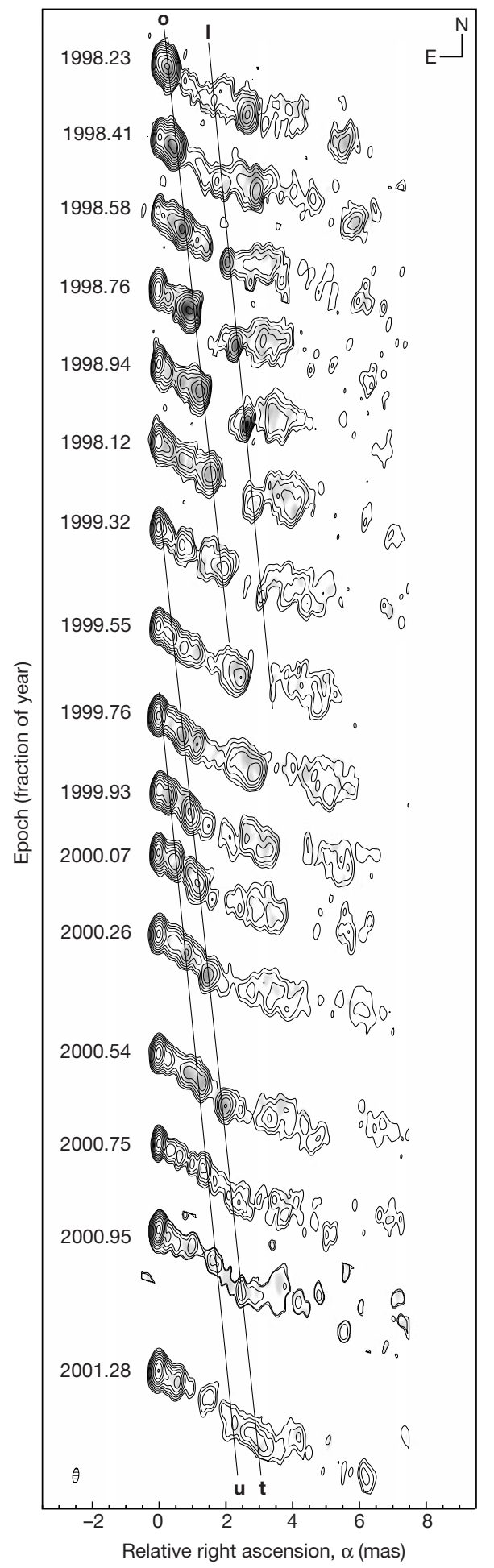

Figure 1 Sequence of VLBA images of $3 \mathrm{C} 120$ at a frequency of $43 \mathrm{GHz}$. Time progresses linearly in the downward direction; for each image, north is upward and east to the left. Contours represent total intensity, with values of $0.5,1,2,4,8,16,32$, 64 and $90 \%$ of the peak of $1.52 \mathrm{Jy}$ per beam (which occurred at epoch 1998.23). Contours increase in intensity towards the centre. Greyscale corresponds to polarized intensity, with a maximum of 36 mJy per beam (at 1998.94). The small ellipse in the bottom left corner is the restoring beam, which approximates the angular resolution in different directions. Diagonal lines trace the approximate path of the superluminal knots, which are identified by the letters $I, 0$, tand $u$, following the sequence of refs 6 and 7 . At the distance of $3 C 120$ of $140 \mathrm{Mpc}$ (for a Hubble constant with value $65 \mathrm{~km} \mathrm{~s}^{-1} \mathrm{Mpc}^{-1}$ ), 1 mas corresponds to a length of $0.70 \mathrm{pc}$ projected on the plane of the sky. 
from other features in the jet by high flux density and significant linear polarization. Their apparent velocities range from 4.1 to $5.0 \mathrm{c}$ (where $c$ is the speed of light, and for a Hubble constant of $\left.65 \mathrm{~km} \mathrm{~s}^{-1} \mathrm{Mpc}^{-1}\right)$. We trace the motions to extrapolated 'ejection' times when each knot was coincident with the 'core', the bright spot on the eastern end of the jet.

We observed 3C120 in the 2.4-20-keV X-ray band from the end of 1997 to early 2000 with the Rossi X-ray Timing Explorer (RXTE). The observations were carried out weekly in 1998 and semi-weekly in 1999, except for 8-week periods when the position of 3C120 was too close to the Sun. To complete the X-ray light curve, shown in Fig. 2a, we processed archival data to add daily RXTE measurements in early 1997. To show the connection between the radio and X-ray emission, we display in Fig. 2 the dates of superluminal ejections as well as the variation with time of the X-ray spectral index $\alpha_{\mathrm{E}}$, total flux density at three radio frequencies, and flux density of the core at $43 \mathrm{GHz}$ (from the images). Each superluminal ejection (with the possible exception of event $t$ ) coincides with the onset of an increase in flux density in at least one of the radio light curves.

We designed our RXTE programme to search for X-ray dips. In order to define such events, we first divide the data into two segments: (A), early 1997; and (B), the remainder. (The MannWhitney test ${ }^{12}$ indicates that the X-ray fluxes from the two sections

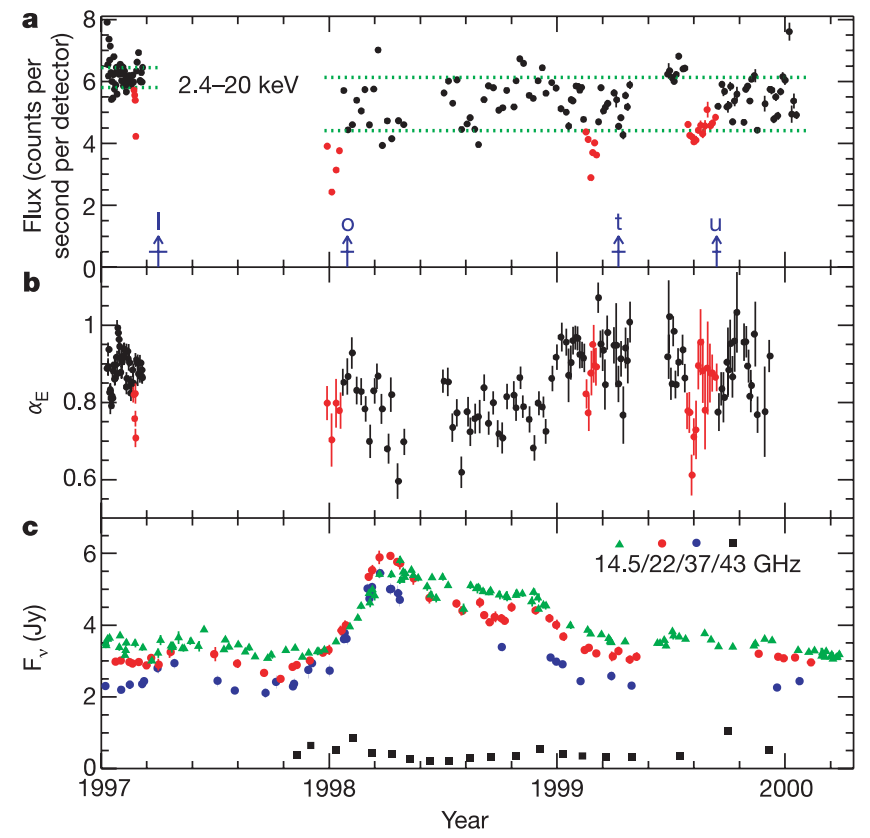

Figure 2 Time dependence of the $\mathrm{X}$-ray and radio emission of 3C120. a, X-ray light curve in photon counts per second per detector; for many data points, the error bars are smaller than the symbols. For each observation, we determined the X-ray flux in photon counts per second over the energy range 2.4 to $20 \mathrm{keV}$ by subtracting an $\mathrm{X}$-ray background model (supplied by the RXTE Guest Observer Facility) from the raw spectrum, using the standard X-ray data analysis software package FTOOLS. We fitted the photon spectrum (observed flux $F_{\text {obs }}$ versus energy $E$ ) with a model consisting of a power-law intrinsic spectrum, $F \propto E^{-\alpha}$, plus photoelectric absorption along the line of sight (the latter is not very important at these energies) using the program XSPEC. Red data points correspond to events that we identify as dips (in $\mathbf{a}$ and $\mathbf{b}$ ), and the green dotted lines delineate $\pm 1 \sigma$ of the mean of each segment of data. Times of ejections of superluminal radio knots are indicated by upward blue arrows, with the horizontal crossbar giving the uncertainty and the labels I, o, t and u corresponding to the features marked in Fig. 1. b, X-ray 'energy' spectral index $\alpha_{\mathrm{E}}$, where $-\alpha_{\mathrm{E}}=\alpha-1$ is the exponent of the power-law dependence of flux density on frequency. c, Radio light curves at $14.5 \mathrm{GHz}$ (green triangles) (University of Michigan Radio Astronomy Observatory); $22 \mathrm{GHz}$ (red circles) and $37 \mathrm{GHz}$ (blue circles) (Metsähovi Radio Observatory); and $43 \mathrm{GHz}$ (core only, black squares) (VLBA). Ref. 6 and this work. are not drawn from the same distribution, with $1.5 \%$ significance.) We identify dips as intervals when $\geqslant 4$ consecutive fluxes lie more than $1 \sigma$ from the mean of the relevant segment of data. We thus identify four dips in the X-ray light curve, denoted in red in Fig. 2a, b.

The Shapiro-Wilk ${ }^{13}$ test shows that the distribution of fluxes from segment $\mathrm{B}$ is consistent with a gaussian distribution. However, this is not the case for segment A unless the outliers (the first dip and the apparent flare near the start of 1997) are removed. In order to test the statistical significance of an observed event consisting of $n$ flux measurements in a data set containing $N$ values, we calculate the probability $P\left(n, N, F_{\mathrm{d}}\right)$ that $n$ consecutive fluxes would fall below a level $F_{\mathrm{d}}$ by random chance, where $F_{\mathrm{d}}$ is the highest point in each dip. We determine the probability $p\left(F \leqslant F_{\mathrm{d}}\right)$ from the best-fit gaussian curve for segment $\mathrm{B}$ and by rank-order (the $i$ th lowest flux out of 52 data points has $p=i / 52$ ) for segment $A$. A recursion formula ${ }^{14}$ gives $P\left(n, N, F_{\mathrm{d}}\right)=0.011,1.2 \times 10^{-3}, 0.010$ and $1.8 \times 10^{-5}$ for the four events. Each event is therefore only very rarely expected to occur by random chance. The significance of each dip is further supported by flattening of the X-ray spectrum (see Fig. 2b), as occurs also in the case of the microquasar GRS $1915+105$ (ref. 15).

It is apparent from Fig. 2 that each X-ray dip is followed shortly by the appearance of a superluminal knot at the site of the radio core. The latter occurred at the following epochs, obtained by a straight line fit to the separation-from-core versus time dependence for each knot: $1997.25 \pm 0.04,1998.08 \pm 0.03,1999.27 \pm 0.03$, and $1999.70 \pm 0.02$. To estimate the probability that the X-ray dips and superluminal ejections are associated by random chance, we consider that there are about two years of X-ray data and a $\sim 0.2$ yr window within which we would consider the events to be related. The probability of any single radio event occurring randomly less than $0.2 \mathrm{yr}$ after a particular X-ray event is thus about 0.1 . There are four events of each type, so the probability of all four radio and Xray events being close in time by chance is thus about $4 !(0.1)^{4} \approx 0.2 \%$. We therefore reject the null hypothesis.

The mean time delay between the minimum in the X-ray flux and the time of ejection is $0.10 \pm 0.03 \mathrm{yr}$. At a typical superluminal apparent speed of $4.7 c$, a knot moves a distance of 0.14 parsecs (pc) in $0.10 \mathrm{yr}$, projected on the plane of the sky. If we adopt an angle of the jet to the line of sight of $20^{\circ}$ (the maximum allowed given the apparent velocity ${ }^{6}$; this minimizes the de-projected distance), the actual distance travelled is $0.41 \mathrm{pc}$. However, it may be the case that the jet starts as a broad wind that accelerates and becomes increasingly focused until it reaches the radio core ${ }^{16-18}$, in which case we calculate that a correction factor of around 0.8 must be applied. We therefore derive a minimum distance of $0.3 \mathrm{pc}$ from the X-ray source to the core of the radio jet. This corresponds to a projected angular displacement of 0.16 milliarcseconds (mas) eastward of the core in the images of Fig. 1. We note that the core of the radio jet is not coincident with the black hole, as has been assumed ${ }^{18}$ in the case of the radio galaxy M87. Rather, it might be where the jet flow reaches its asymptotic relativistic velocity ${ }^{16}$ or the site of standing shocks that accelerate electrons to ultrahigh energies ${ }^{5,19}$. This result implies that the jet might be essentially invisible between its site of origin near the black hole ${ }^{3}$ and the radio core far downstream where its plasma is re-energized.

The correlated X-ray and radio behaviour of $3 \mathrm{C} 120$ is similar to that of the microquasar GRS1915+105: X-ray dips during which the spectrum hardens, each followed by the ejection of a bright superluminal radio knot. This phenomenon has been interpreted ${ }^{1,4}$ as resulting from an instability in the accreting flow causing a piece of the inner disk to break off. Part of this matter is drawn into the event horizon of the black hole but with considerable matter and energy ejected down the jet. The loss of some of the inner disk causes a drop in the soft X-ray flux, which is observed as a dip plus hardening of the spectrum. The hard X-ray flux, presumably the product of 
Compton scattering-for example, in a corona above the disk ${ }^{1,411}$ is not affected as much. According to this picture, the time delay between the X-ray dip and the appearance of the superluminal knot corresponds to the travel time of the disturbance from the inner accretion disk to the core of the radio jet.

Another property shared by $3 \mathrm{C} 120$ and GRS1915+105 is the bulk velocity of the jet needed to explain the apparent superluminal motion: $0.98 c$ in each case (Lorentz factor $\approx 5)^{6,20}$. The one-sided observed jet in 3C120 contrasts with the two oppositely directed jets in the microquasar. This can readily be explained as a consequence of the stronger relativistic beaming of the approaching jet in $3 \mathrm{C} 120$, because the angle between the jet and the line of sight is smaller $\left(<20^{\circ} \text { compared with } 66^{\circ} \text { in GRS1915+105 }\right)^{6,20}$.

The radio galaxy $3 \mathrm{C} 120$ is therefore similar to a scaled-up version of a microquasar. It may be possible to adapt models for the closer microquasars-whose accretion processes are better studied owing to higher and more rapidly variable X-ray fluxes-to their distant AGN cousins. We expect the scaling factor to be proportional to the radius of the innermost orbit around the black hole ${ }^{3}$, which ranges from $3 R_{\mathrm{S}}$ for a static black hole to $0.5 R_{\mathrm{S}}$ for the maximally rotating case $^{21}$. Here, $R_{\mathrm{S}} \propto M$ is the Schwarzschild radius, with the mass of the black hole $M=1.6-5.1 \times 10^{7}$ solar masses in 3C120 (ref. 22) and 10-14 solar masses in GRS1915+105 (ref. 2). The distance of the core of the radio jet to the black hole is about $7 \times 10^{4}$ times higher in 3C120 than in GRS1915+105 (ref. 20), whereas the time interval between $\mathrm{X}$-ray dips is about $5 \times 10^{5}$ times longer $(0.5-1.2 \mathrm{yr}$ for $3 \mathrm{C} 120$ versus $25-100 \mathrm{~s}$ for the microquasar $\left.{ }^{4}\right)$. These values are, respectively, 20-70 and 2-10 times lower than the ratio of black hole masses. The factor of $2-10$ is small compared to the uncertainties in our theoretical understanding of how the time between events relates to the physical parameters of the accretion disk. The greater discrepancy in the ratio of length scales implies that the conditions governing formation of the radio core-perhaps the pressure of the external medium or the magnetic field in the jet-are different in the two objects.

Received 15 January; accepted 26 April 2002; doi:10.1038/nature00772.

1. Mirabel, I. F. \& Rodríguez, L. F. Microquasars in our Galaxy. Nature 392, 673-676 (1998)

2. Greiner, J., Cuby, J. G. \& McCaughrean, M. J. An unusually massive stellar black hole in the Galaxy. Nature 414, 522-525 (2001).

. Meier, D. L., Koide, S. \& Uchida, Y. Magnetohydrodynamic production of relativistic jets. Science 291, 84-92 (2000).

. Belloni, T. Inner disk oscillations. Astrophys. Space Sci. 276 (suppl.), 145-152 (2001).

5. Gómez, J. L., Martí, J. M., Marscher, A. P., Ibáñez, J. M. \& Alberdi, A. Hydrodynamical models of superluminal sources. Astrophys. J. 482, L33-L36 (1997).

6. Gómez, J. L., Marscher, A. P., Alberdi, A., Jorstad, S. G. \& García-Miró, C. Flashing superluminal components in the jet of the radio galaxy 3C120. Science 289, 2317-2320 (2000).

7. Gómez, J. L., Marscher, A. P., Alberdi, A., Jorstad, S. G. \& Agudo, I. Monthly 43 GHz VLBA polarimetric monitoring of $3 \mathrm{C} 120$ over 16 epochs: evidence for trailing shocks in a relativistic jet. Astrophys. J. 561, L161-L164 (2001).

8. Grandi, P. et al. ROSAT, ASCA, and OSSE observations of the broad-line radio galaxy 3C 120. Astrophys. J. 487, 636-643 (1997).

9. Halpern, J. P. X-ray spectrum and variability of 3C 120. Astrophys. J. 290, 130-135 (1985).

10. Maraschi, L. et al. Coordinated X-ray, ultraviolet, and optical observations of 3C 120. Astrophys. J. 368, 138-151 (1991).

11. Zdziarski, A. \& Grandi, P. The broadband spectrum of 3C 120 observed by BeppoSAX. Astrophys. J. 551, 186-196 (2001)

12. Mann, H. B. \& Whitney, D. R. On a test of whether one of two random variables is stochastically larger than the other. Ann. Math. Stat. 18, 50-60 (1947).

13. Shapiro, S. S. \& Wilk, M. B. An analysis of variance test for normality (complete samples). Biometrika 52, 591-611 (1965).

14. Feller, W. An Introduction to Probability Theory and its Applications, 2nd edn 322 (Wiley, New York, 1968)

15. Klein-Wolt, M. et al. Hard X-ray states and radio emission in GRS 1915+105. Mon. Not. R. Astron. Soc. 331, 745-764 (2002).

16. Marscher, A. P. Relativistic jets and the continuum emission in QSOs. Astrophys. J. 235, 386-391 (1980).

17. Reynolds, S. P. Theoretical studies of compact radio sources. I. Synchrotron radiation from relativistic flows. Astrophys. J. 256, 13-27 (1982).

18. Junor, W., Biretta, J. A. \& Livio, M. Formation of the radio jet in M87 at 100 Schwarzschild radii from the central black hole. Nature 401, 891-892 (1999).

19. Daly, R. D. \& Marscher, A. P. The gas dynamics of compact relativistic jets. Astrophys. J. 334, 539-551 (1988).

20. Fender, R. P. et al. MERLIN observations of relativistic ejections from GRS 1915+105. Mon. Not. R. Astron. Soc. 304, 865-876 (1999).
21. Shapiro, S. I. \& Teukolsky, S. A. Black Holes, White Dwarfs and Neutron Stars: the Physics of Compact Objects 362 (Wiley Interscience, New York, 1983).

22. Wandel, A., Peterson, B. M. \& Malkan, M. A. Central masses and broad-line region sizes of active galactic nuclei. I. Comparing the photoionization and reverberation techniques. Astrophys. J. 526, 579-591 (1999).

\section{Acknowledgements}

We gratefully acknowledge partial support for this research from the US National Science Foundation (NSF), NASA, the Fulbright Commission for collaboration between Spain and the USA, and the Spanish Dirección General de Investigación Científica Técnica. The VLBA is an instrument of the National Radio Astronomy Observatory, a facility of the NSF operated under cooperative agreement by Associated Universities Inc. The University of Michigan Radio Astronomy Observatory was funded in part by the NSF and by the University of Michigan Department of Astronomy.

\section{Competing interests statement}

The authors declare that they have no competing financial interests.

Correspondence and requests for materials should be addressed to A.P.M. (e-mail: marscher@bu.edu).

\section{Coherence-incoherence and dimensional crossover in layered strongly correlated metals}

\section{T. Valla*, P. D. Johnson*, Z. Yusof $\dagger$, B. Wells $\dagger$, Q. Li $\neq$, S. M. Loureiro $\$$ R. J. Cava $\varsigma$, M. Mikami||, Y. Mori||, M. Yoshimura\| \& T. Sasaki\|}

* Physics Department, $\neq$ Material Sciences Department, Brookhaven National Laboratory, Upton, New York 11973, USA

$\dagger$ Department of Physics, University of Connecticut, 2152 Hillside Road U-46, Storrs, Connecticut 06269, USA

$\$$ Department of Chemistry and Princeton Materials Institute, Princeton University, Princeton, New Jersey 08540, USA

|| Department of Electrical Engineering, Osaka University, 2-1 Yamada-oka, Suita-shi, Osaka, 565-0871 Japan

The properties of an interacting electron system depend on the electron correlations and the effective dimensionality. For example, Coulomb repulsion between electrons may inhibit, or completely block, conduction by intersite electron hopping, thereby determining whether a material is a metal or an insulator ${ }^{1}$. Furthermore, correlation effects increase as the number of effective dimensions decreases; in three-dimensional systems, the low-energy electronic states behave as quasiparticles, whereas in one-dimensional systems, even weak interactions break the quasiparticles into collective excitations ${ }^{2}$. Dimensionality is particularly important for exotic low-dimensional materials where one- or two-dimensional building blocks are loosely connected into a three-dimensional whole. Here we examine two such layered metallic systems with angle-resolved photoemission spectroscopy and electronic transport measurements, and we find a crossover in the number of effective dimensions-from two to three-with decreasing temperature. This is apparent from the observation that, in the direction perpendicular to the layers, the materials have an insulating character at high temperatures but become metal-like at low temperatures, whereas transport within the layers remains metallic over the whole temperature range. We propose that this change in effective dimensionality correlates with the presence of coherent quasiparticles within the layers.

Crossover in interlayer transport has been detected in layered metals such as $\mathrm{Sr}_{2} \mathrm{RuO}_{4}$ (ref. 3) and $\mathrm{NaCo}_{2} \mathrm{O}_{4}$ (ref. 4), and more recently in $\left(\mathrm{Bi}_{1-x} \mathrm{~Pb}_{x}\right)_{2} \mathrm{M}_{3} \mathrm{Co}_{2} \mathrm{O}_{y}$ (where $\mathrm{M}$ is $\mathrm{Ba}$ or $\left.\mathrm{Sr}\right)^{5,6}$. The layers appear 'isolated' at high temperatures, but become connected at low 